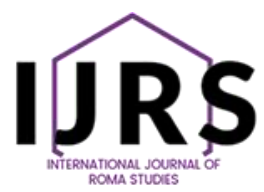

Hipatia Press

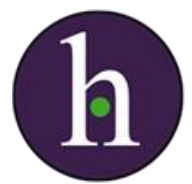

Instructions for authors, subscriptions and further details:

http://ijrs.hipatiapress.com

\title{
Del prácticum presencial al prácticum on-line, en la formación inicial de maestras y maestros durante la pandemia del COVID-19, en contextos de exclusión social
}

Consol Aguilar Ródenas ${ }^{1}$

1) Universitat Jaume I, Spain

Date of publication: March $15^{\text {th }}, 2021$

Edition period: March 2021 - July 2021

To cite this article: Aguilar Ródenas, C. (2021). Del prácticum presencial al prácticum on-line, en la formación inicial de maestras y maestros durante la pandemia del COVID-19, en contextos de exclusión social. International Journal of Roma Studies, 3(1), 73-96. doi: 10.17583/ijrs.2021.6386

To link this article: http://dx.doi.org/10.17583/ijrs.2021.6386

PLEASE SCROLL DOWN FOR ARTICLE

The terms and conditions of use are related to the Open Journal System and to Creative Commons Attribution License (CCAL). 


\section{From face-to-face prácticum to online prácticum in the initial training of teachers during the COVID-19 pandemic, in contexts of social exclusion}

Consol Aguilar Ródenas

Universitat Jaume I

\section{Abstract}

We must reflect on what has happened during lockdown, take into account the leading voices of our student body in order to improve highlighting what has not worked out. This paper collects the voices of 20 students who begin their face-toface initial teacher training practices and, from lockdown, go on to do them virtually. Of this total, 10 were in the third-year initial training practices and 10 in the fourth one. The centers are four public Pre-school and Primary Education centers in Castelló, 3 of them Specific Action Centers, and 1 center that receives students from late schooling and unschooling. The first three are predominantly Roma. The fourth hosts the course that concerns us of late schooling or unschooling students from 17 countries. Being aware of what has happened in these specific educational contexts is urgent so that all future regulations include them, do not become invisible and their specific contexts are taken into account.

Keywords: Student voices; Teacher Training; Practices; Pandemic; Digital, Social And Educational Gap. 


\section{Del prácticum presencial al prácticum on-line, en la formación inicial de maestras y maestros durante la pandemia del COVID-19, en contextos de exclusión social}

Consol Aguilar Ródenas

Universitat Jaume I

\section{Resumen}

Debemos reflexionar sobre lo que ha ocurrido durante el confinamiento, tener en cuenta las voces protagonistas de nuestro estudiantado para mejorar a partir de la visibilización de lo que no ha funcionado. Este artículo recoge las voces de 20 estudiantes que empiezan sus prácticas de formación inicial de maestro-a presenciales y, a partir del confinamiento, pasan a realizarlas de manera virtual. De este total 10 estaban en las prácticas de formación inicial de tercer curso y 10 en las de cuarto curso. Los centros son cuatro CEIP públicos de Castelló, 3 de ellos CAES (Centros de Acción Específica) y 1 centro que recibe alumnado de escolarización sobrevenida. Los tres primeros son mayoritariamente de población gitana. El cuarto acoge el curso que nos ocupa alumnado de escolarización sobrevenida durante todo el curso, con alumnado procedente de 17 países. Conocer lo que ha ocurrido en estos contextos educativos específicos es urgente para que todas las normativas futuras los incluyan, no se invisibilicen y sus contextos específicos se tengan en cuenta.

Palabras clave: voces del estudiantado; formación del profesorado; practicas; pandemia; brecha digital, social y educativa

2021 Hipatia Press

ISSN: $2462-425 \mathrm{X}$

DOI: $10.17583 / \mathrm{ijrs} .2021 .6386$

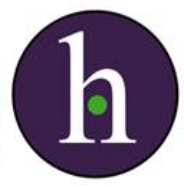


i queremos mejorar el futuro, deberemos reflexionar sobre el presente, atender a las investigaciones educativas que se han realizado en el periodo del coronavirus y escuchar a todas las personas de la comunidad educativa. Este artículo recoge las voces de 20 estudiantes de la titulación de Maestra-o de la Universitat Jaume I, durante el complejo periodo de su prácticum en los colegios el curso 2019-2020, hay que recuperar las voces del estudiantado para que, en primera persona cuenten lo que han vivido y cómo lo han vivido, compartir lo que vivimos en pandemia en el prácticum.

De un día para otro tuvimos que reorganizar la docencia con actividades on-line cuando fuera posible. El estudiantado afectado en España fueron 10 millones de estudiantes, 8’5 millones en educación no universitaria (Diez \& Gajardo, 2020a, p. 103).

Ya tenemos algunas respuestas que evidencian lo que ha pasado. Diez y Gajardo a partir de una investigación a partir de una encuesta a la que han respondido 3.400 familias y estudiantes de toda España durante los meses de marzo y abril de 2020, muestran que los resultados ofrecen cinco ideas fundamentales (Diez \& Gajardo, 2020a): 1) la brecha digital se suma y amplifica la brecha social, aumentando la desigualdad en épocas de crisis; 2) las tecnologías, que han sido un aliado esencial en la crisis, no son una alternativa a la relación educativa presencial, pero son una herramienta cada vez más enriquecedora para apoyar el proceso de enseñanza-aprendizaje; 3) es necesario repensar el actual currículum enciclopédico para discriminar lo prescindible de lo imprescindible y priorizar los contenidos relevantes; 4) evaluar no es calificar, es entender cómo ha sido el proceso de aprendizaje para ayudar a cada alumno y alumna a seguir avanzando, y 5) es necesario "cuidar a las personas" y primar su bienestar integral, para "que nadie se quede atrás".

Un estudio del Proyecto Atlántida realizado con 3.700 profesionales de la enseñanza, sobre cómo se han desarrollado los aprendizajes en las semanas del confinamiento muestra que un tercio de las chicas y los chicos no pudieron seguir el aprendizaje a distancia. Un 25\% de las familias (5.900) reconocieron distintas clases de dificultades para que sus hijas e hijos pudieran seguir aprendiendo adecuadamente (Redacción, 2020). Hay que recordar que muchas familias han tenido que compartir ordenadores u otros dispositivos digitales a causa del teletrabajo desde sus casas, compaginando 


\section{Aguilar-Practicum on-line formación profesorado COVID-19}

además con los cuidados familiares. Aparte de este aspecto de infraestructura o materiales, el estudio del Proyecto Atlántida subraya la falta de formación de familias, alumnado y docentes en el uso de las TIC, "en la creación de espacios que pudieran ser homologables a un aula".

Cerrar los centros ha supuesto que, en una tercera parte de los casos, la pérdida de aprendizaje ha podido ser severa (Redacción, 2020). El impacto del confinamiento en el aumento de la segregación también lo confirma una encuesta al profesorado sobre como afrontaban el confinamiento, de las profesoras de Pedagogía Aina Tarabini y Judith Jacoukis a 2.777 profesorases que revela que su mayor preocupación $\left(81^{\prime} 5 \%\right)$ era el bienestar emocional y personal de su alumnado y, en segundo lugar (77’6\%) el acompañamiento de las familias (Rius, 2020). Otro estudio realizado entre Educación Conectada, FAD y BBVA, elaborado a partir de los datos de cuestionarios a profesorado, familias y alumnado, revela la sensación de abandono que tienen, desde el inicio del estado de alarma, los más de 5000 profesores-as que han contestado a la encuesta y que han participado en los grupos de discusión de la investigación con "respuestas superficiales por parte de las administraciones para hacer frente a la formación a distancia" (Gutierrez, 2020a), señalando que han tenido que afrontar una nueva modalidad educativa, con sus propios equipos.

Todas las investigaciones subrayan la necesidad de inversión, la necesidad de formación docente, la necesidad de reducir la ratio aumentando la contratación de profesorado y modificando espacios escolares, el replanteamiento del currículum escolar y la necesidad de dotar de recursos para usar las TIC como apoyo y no como sustitución de la presencialidad (Díez \& Gajardo, 2020b).

Luis Torrego (2020) subraya que la desigualdad es anterior a la pandemia con un millón y medio de menores en situación de pobreza severa, España es el segundo país de la UE en fracaso escolar siempre ligado a los grupos más vulnerables, destacando que el sistema educativo no es equitativo $\mathrm{y}$, además, es un mecanismo de discriminación social, destacando: el conformismo de la opinión pública con la desigualdad, su "naturalización"; la transferencia de dinero público a la concertada que no acoge con igualdad a todas las clases sociales y el marketing educativo con todo lo que conlleva que ha hecho retroceder la buena educación, la que convierte las dificultades en posibilidades, la que incluye a todas y a todos y no se deja a nadie detrás. 
En la pandemia se incrementó la pobreza en el alumnado de familias más vulnerables y con menos recursos (1 de cada 4 niños y niñas que viven en España (EAPN. 2020), como evidencia la desconexión digital en el mundo rural, más la brecha digital de segundo orden (es decir, el distinto uso de la tecnología según la clase social) (Diez \& Gajardo, 2020a). Hay que tener en cuenta que la brecha digital y social existente no aparece con la pandemia, ignorarlo es desconocer el estado de la cuestión en España.

Como señala Juana Sancho (2020): "Me han sorprendido quienes afirman que la pandemia ha revelado los estragos de la desigualdad, la pobreza, las brechas sociales, tecnológicas, educativas y sanitarias. De verdad no lo sabían?. Si son educadores, en qué mundo vivían?’.

La situación se agrava en el contexto del prácticum objeto de este estudio. Diaz y Gajardo (2020a) subrayan una realidad que para la mayoría de las niñas y los niños de los colegios donde el estudiantado de este artículo hacía su prácticum ha sido su contexto:

Buena parte de las familias con más necesidades "carece de las condiciones materiales (tecnología1, conexión a la red, espacio, temperatura, luz, etc.), las herramientas culturales (habilidades pedagógicas, conocimiento del idioma, formación, etc.), el tiempo para acompañar el proceso educativo, la estabilidad emocional (por problemas económicos, de salud, habitacionales, etc.) o los recursos alimentarios necesarios para aprender" (Martín \& Rogero, 2020), así como su "capital cultural" y su "lenguaje académico" están más distantes a la cultura escolar académica tradicional (Alonso, 2019; Martín Criado, 2019; 2020; Martín Criado \& Gómez Bueno, 2017). No se puede esperar que el curso siga sin más, porque muchas familias siguen sin tener acceso, hoy en día, a los mínimos vitales que le permitan su subsistencia, viviendo hacinadas en infraviviendas con una situación de estrés difícilmente imaginable (Makarov \& Lacort, 2020). (p. 106)

El gobierno valenciano envió a los centros y equipos docentes las instrucciones de cómo debían desarrollar el proceso educativo en el contexto del confinamiento centrado en las competencias básicas y una evaluación formativa $\mathrm{y}$, como todas las comunidades autónomas, remarcando la necesidad de cumplir con los derechos incluidos en la Convención de los Derechos de la Infancia de la ONU. Se recomendó repasar, reforzar y consolidar los aprendizajes de los dos primeros trimestres del curso y que, de 


\section{Aguilar-Practicum on-line formación profesorado COVID-19}

manera general, no se avanzara en nuevos contenidos (Diez \& Gajardo, 2020a).

Pablo Gutiérrez (2020a) recuerda que Save the Children en su informe sobre la situación de la pandemia en el que aborda los problemas más importantes del sistema educativo, reclama 4.200 millones para el sistema educativo, entre otros aspectos contratando más personal de apoyo para que nadie se quede atrás. La Organización de Consumidores y Usuarios (OCU) ha realizado un estudio a partir de los datos de 903 familias con hijos-as en Educación Primaria que revela que en los colegios públicos "apenas el 62 por ciento de los alumnos" ha disfrutado de clases online por videollamada con los/las profesores. En los colegios concertados estas clases online con videollamada han llegado al 84 por ciento. Es decir, uno de cada tres niñosas de Primaria no recibió clases online durante el confinamiento, que dejó a 9,5 millones y medio de estudiantes en España sin clases presenciales. Otras alternativas de enseñanza online señaladas a partir de este estudio muestran que, en el confinamiento, han sido: el envío de vídeos realizados por los propios profesores (30\% entre los alumnos de colegios públicos; $42 \%$ en concertados), el envío de tareas para descargar, realizar y devolver luego a los profesores $(66 \%$ de los alumnos de colegios públicos y $78 \%$ de concertados) o el uso de plataformas virtuales para realizar ejercicios $(37 \%$ en los públicos; $55 \%$ en concertados).Respecto a la satisfacción final de los padres/madres con la educación online es de 6,1 sobre 10 porque el 38 por ciento de los niños-as tenían dificultades para concentrarse durante las videollamadas o a la hora de atender a los vídeos enviados por los profesores, necesitando la ayuda de las familias (Agencia Europa Press, 2020).

Enrique Diez (Diez \& Torrego, 2020), reivindica presupuestos escandalosos para cuidar la vida en común, el 7\% del PIB, pasar de 40 mil millones a 70 millones, al menos lo mismo que gastamos en armamento. Como evidencia Enrique Diez (2020b) existe tolerancia a la desigualdad y la injusticia desde la construcción de un consenso interesado para "olvidar todo conocimiento relativo al contexto y la estructura y, por supuesto, su participación en la producción de la pobreza y las injusticias sociales”. Diez defiende la necesidad de que el plan de reconstrucción educativa inclusiva, incluya bajar la ratio y aumentar la plantilla docente, afirmando que la revolución educativa pendiente no es tecnológica, es inclusiva. Estos 
cambios deberían ir unidos, destaca, de una reducción de la fragmentación curricular en asignaturas seleccionando el contenido relevante, el trabajo coordinado y en equipo del profesorado, la participación de las familias y unas condiciones laborales dignas, entre otras necesidades.

\section{Nuestro contexto}

El estudiantado de formación inicial de Maestra-o del prácticum objeto de este artículo está integrado por 20 estudiantes: 10 mujeres en PI (prácticum de tercero), 5 mujeres en PII (prácticum de cuarto) y 5 hombres en PI. Del total 6 estudiantes estaban en EI y 14 en EP.

Las maestras y maestros supervisores fueron 20 (17 maestras y 3 maestros). Repartidos en 15 en PI (13 maestras y 2 maestros) y 5 en PII (4 maestras y 1 maestro).

Los centros son cuatro CEIP públicos de Castelló, 3 de ellos CAES (Centros de Acción Específica)- uno de ellos Comunidad de Aprendizaje (en adelante CdA)- y 1 centro que recibe alumnado de escolarización sobrevenida. Los tres primeros son de población gitana unida (aparece muy puntualmente alumnado sudamericano, africano o de países del este de Europa), mayoritariamente, a la exclusión social. El cuarto acoge el curso que nos ocupa alumnado de escolarización sobrevenida durante todo el curso y su alumnado procede de 17 países (5 africanos, 7 europeos- incluyendo alumnado gitano-, 3 asiáticos y 2 sudamericanos).

El PI se debía desarrollar en los colegios desde el 26 de febrero hasta el 22 de mayo. El PII desde el 22 de enero hasta el 22 de mayo. El 14 de marzo de 2020 se decretó, como consecuencia de la pandemia del COVID-19, el estado de alarma en España y se cerraron todos los centros educativos. A partir de ahí el prácticum pasó de presencial a virtual, en un contexto de pandemia global. Son los futuros-as maestros y maestras, una identidad profesional que no podemos difuminar en otros conceptos, en otros contextos.

\section{El prácticum presencial}

El estudiantado comenzó su prácticum como cada curso escolar. Desde el momento que eligen colegios ligados a la exclusión social ya hacen una 


\section{Aguilar-Practicum on-line formación profesorado COVID-19}

opción. Acordamos que realizaran diarios dialógicos, reflexionando sobre todo lo que vivían, sobre la realidad de su contexto (Aguilar, 2012).

La mayoría del estudiantado manifiesta nerviosismo ante su primer día de prácticum en las escuelas. En sus diarios encontramos que una estudiante argumenta:

Han aparecido muchas dudas (...) sobre si seré o no seré capaz de ser una buena maestra y estar a la altura de mi futuro alumnado y de la sociedad. Llevo queriendo ser maestra desde que tengo uso de razón y hoy que he estado delante de un aula, me he sentido realmente pequeña.

Otra escribe: "Muchas incógnitas me rondaban como, por ejemplo, el curso que me tocaría, como sería mi tutora o si sería capaz de conectar con el alumnado y hacerlo bien". Y otra estudiante expresa: "muchas incógnitas y dudas me rondaban como, por ejemplo, el curso que me tocaría o si sería capaz de conectar con el alumnado y hacerlo bien". Y una estudiante de más edad escribe:

A la entrada del colegio me reúno con mis compañeros y compañeras de prácticas y puedo observar sus expresiones de ilusión, nervios e inquietud. Creo que puedo aprender mucho de ellos porque son más jóvenes, sin experiencia previa y, por tanto, puedo observar en ellos aspectos que quizás yo no sea consciente y se me pasen por alto y que resulten muy positivos para mi aprendizaje.

Uno de los estudiantes expresa:

Cuando escogimos las prácticas, a la mayoría de nosotros nos aconsejaban o nos decían que era mejor haber hecho otra elección y nos daban sus opiniones (...) repletas de prejuicios, ya que nunca habían estado en dicho barrio. Nosotros mismos, al empezar las prácticas llegamos con muchos prejuicios y miedos, como que nos robaran, nos reyaran el coche o tener que medir las palabras que usábamos respecto a temas como el luto y la muerte.

Procedente del entorno rural, una de sus compañeras, percibe la cercanía del espacio físico y reflexiona: "El barrio me ha recordado bastante a un pueblo, ya que son todo casas de poca altura, dentro de él, parecía que estuviéramos en un pueblo cercano, independiente de la ciudad". 
Es un contexto muy diferente a la realidad que han vivido hasta entonces que les impacta, ya no son contextos del telediario o la prensa, son personas reales afectadas por ejemplo por las migraciones producidas por las guerras. Y desconocen estos contextos educativos, estigmatizados injustamente. Una de las estudiantes expresa sobre una niña:

Me impactó muchísimo saber que vino de Siria a España con su madre y su hermana, desafortunadamente su padre falleció, allí en la guerra de su país. Al conocer esta historia no sabía cómo sentirme (...) comprendí que ahora estaba en un mundo mucho más auténtico.

Recoge en su diario dialógico otra compañera que un niño le había comentado que dos días antes había sido el cumpleaños de su madre, y cuenta:" le he preguntado si lo había celebrado comiendo tarta. Me respondió que no pudo, porque su madre está en la cárcel. Ha sido uno de los momentos más duros". Esta diversidad de contextos educativos, aparecen invisibilizados en su formación inicial de maestras y maestros. Otra estudiante se sorprende de la falta de higiene:" la mayoría de ellos gasta la misma ropa para venir a clase muchos días (...) Llegan al aula sin duchar". Es el inicio del prácticum y todavía no han profundizado en las causas. Pero quieren hacerlo como se aprecia en el texto de otra estudiante que muestra que desea conocer estas otras realidades a las que no está acostumbrada:

Es una aventura que te permite abrir tu mente, cambiar tu mirada, no juzgar, ver más allá de lo que se ve a simple vista y dejar atrás muchos prejuicios .Yo tenía claro que quería venir a esta escuela, pero tenía miedos e inseguridades respecto a ella, el alumnado o el barrio, y poco a poco he ido dejándolo atrás y superándolo.[...]las practicas me han ayudado a tener todavía más claro el tipo de maestra que quiero ser, una que no los juzgue, que los ayude, tanto a aprender como a respetarse, quererse y hacer lo mismo con el resto. Quiero que aprendan y disfruten, mientras lo hacen, verlos madurar, crecer, reflexionar y empatizar con la gente que tienen a su alrededor. Como futura maestra creo que es imprescindible recordarnos que tenemos en nuestras manos a los adultos de dentro de 20 años.

La función de la escuela en este contexto es muy importante. Una estudiante destaca: 
Este colegio lucha por la inclusión, la colaboración y la comunicación entre la escuela, el barrio y las familias (...) ya que los niños no conocen más allá del barrio, de su casa y la plaza, se quiere que los niños vean más allá y conozcan y aprendan cosas nuevas.

También reflexionan sobre la maestra supervisora:

Un aula es un espacio muy personal e íntimo. Recibir a alumnos/as en prácticas es como invitar a tu casa a alguien que no conoces en absoluto para que se quede durante una temporada viviendo junto a ti y aprendiendo de ti y considero que, esto, es de agradecer (...) Me ha dado mucha libertad y poder de decisión y eso me ha transmitido mucha confianza (...) Esa confianza me calma y me transmite seguridad

Para enriquecerse mutuamente desde la reflexión, una vez por semana se reunía todo el estudiantado de prácticum en cada colegio para hacer una Tertulia Pedagógica Dialógica (TPD) sobre un artículo científico (Aguilar, 2017). El agrupamiento de estudiantes por colegio que integraba cada TPD era: 5, 6, 6 y 3. Eran reuniones inter-nivelares que enriquecían el prácticum. Un estudiante destaca sobre las reuniones inter-nivelares: "Si no las hubiéramos hecho, si no nos hubiéramos comunicado tanto durante este periodo, me habría perdido una gran cantidad de aprendizajes nuevos, vivencias, inquietudes y dificultades de mis compañeras".

Además, se reunían individualmente con su maestro-a supervisor-a, con quien dialogaban sobre el contenido de su diario dialógico. También se celebraron reuniones tanto conmigo (su tutora), como conjuntas en las que participamos todas las personas implicadas en el prácticum. Sobre las TPD que han realizado durante el prácticum presencial, señalan desde el resumen de una de las tertulias:

Las tertulias son una buena práctica para desarrollar nuestro espíritu crítico, una cualidad que todo buen docente debería tener para seguir creciendo personal y profesionalmente. Así como el saber argumentar y escuchar las opiniones y las propuestas de los demás.

Una estudiante en la TPD descubre que Ausubel era racista y clasista y expresa: "Considero que no es nada lógico que haya asignaturas de grado 
que defiendan la educación en la diversidad y, al mismo tiempo, defiendan teorías que excluyen esa misma diversidad". Y una de las estudiantes, que hace su prácticum en una CdA, además participa en una TPD con las familias sobre un artículo sobre violencia infantil, queda fascinada por todo lo que ocurre, por los comentarios de las familias, que rompen muchos de sus prejuicios. En otro colegio argumentan:

Sin ninguna duda, (...) la TPD ha funcionado y nos ha permitido compartir experiencias, ideas, preocupaciones, además, de empatizar con aquello que están viviendo otros compañeros-as. [...] Pensamos que es una manera de escucharnos los unos a los otros de manera activa y ayudarnos mutuamente que también tendremos que hacer el día de mañana como equipo interdisciplìnar cuando ejerzamos.

Los diarios dialógicos habían ayudado a reflexionar sobre el contexto. Una de las estudiantes expresa su transformación:

He podido observar una evolución positiva: el gran cambio que he tenido a la hora de expresar lo que quería transmitir. Día a día he observado que tanto mis pensamientos como mi mirada ya se fijaban en otros aspectos que, en un primer momento, podían parecer poco interesantes pero que, después, les sacas mucho jugo (...) mi conocimiento se ha ido transformando y se han ido reconstruyendo mis ideas de una manera más crítica (...) todo gracias a las reflexiones diarias, a los intercambios de opinión con mis compañeros (...) A través del diálogo se puede compartir y aprender mucho todos juntos.

Un ejemplo de la reflexión que une trabajo en la escuela, diarios dialógicos y TPD son las reflexiones que realizan sobre el género. El contexto de la mayoría de familias en los tres CAES es sexista. En la cuarta escuela influyen los contextos culturales diversos. Persiste la idea de que el espacio privado es el de la mujer y el hombre es el encargado de llevar dinero a casa. Este universo binario se plasma en la vida cotidiana. Una estudiante explica que una alumna "comenta que sus padres piensan que no tiene que venir más a clase, porque ya tiene los conocimientos suficientes para leer, y que es mejor que se quede en casa fregando". Otra estudiante recoge que en su clase de quinto de primaria "hemos estado arreglando y recogiendo el aula que estaba bastante desordenada. Ha habido un comentario que me ha 


\section{Aguilar-Practicum on-line formación profesorado COVID-19}

chocado mucho y es que, uno de los alumnos, le ha dicho a otra alumna: "tú que eres chica, coge la escoba y barre". La estudiante señala que le ha sorprendido que la niña, con mucho carácter, aceptara. La maestra y ella intervienen y al final, otro compañero decide barrer. La estudiante señala que esa actitud está normalizada en sus casas y concluye que espere que la escolarización les ayude a transformar su mirada. Otro de los estudiantes reflexiona que es un tema delicado porque debe respetarse la cultura de las familias, argumentando:

Tenemos la obligación y la necesidad de crear entornos de respeto y de igualdad dentro del ámbito escolar (...) para que desde la docencia [se]pueda en algún momento crear una buena base de transformación y, a lo mejor, cambiar mínimamente esa percepción sexista de la vida.

La relación con el maestro-a supervisor-a es muy importante. Una estudiante explica cómo ha sido su relación con su maestra: "ha sido maravillosa. Desde el primer día que estuve en el centro, ha estado pendiente de mí, de mis dudas, inquietudes... e integrándome en la clase y el centro hasta conseguir que fuera una más".

Una de las estudiantes que realiza su prácticum en una $\mathrm{CdA}$ expresa sobre su maestra supervisora:

En las diversas situaciones que han surgido en el día a día del aula creo que he aprendido mucho de su forma de tratar, ver y solucionar los conflictos. Su forma de promover el diálogo en el aula y su habilidad para no señalizar los errores y felicitar los aciertos, haciendo que sean los propios niños y niñas los que intenten promover el comportamiento positivo, evitando así dar protagonismo a los que realizan comportamientos negativos, Una de las cosas que me llamó la atención es (...) "el club dels valents"(...) ayuda mucho a los alumnos-as y promueve muy bien el buen comportamiento, hace que se expresen y dialoguen ayudando a sus compañeros a poder mejorar.

Las transformaciones que han vivido como protagonistas en el mismo centro las destaca una compañera:

Me he dado cuenta de que son posibles las experiencias pedagógicas en las que el diálogo, la participación activa de la familia y de la comunidad educativa son el eje fundamental de la educación. Y también se observan 
mejoras de los resultados académicos, vivencia de valores entre el alumnado $\mathrm{y}$ transformaciones a nivel social y cultural del entorno que convierten el centro en un referente para las familias de los alumnos. Este colegio debería ser un modelo para otras escuelas, porque nos demuestra que, con la implicación de maestros, alumnos y de sus familias se puede llegar muy lejos.

\section{El prácticum en el confinamiento}

Cuando se decreta el confinamiento, el estudiantado de PI lleva dos semanas de prácticum presencial y el de PII siete semanas. El último día de clase antes del confinamiento, un estudiante escribe en su diario dialógico:

Tengo sentimientos de frustración, rabia y miedo por tener que parar esta etapa, y además no saber si podremos volver o cuando volveremos. A pesar de todo y viendo el nivel de propagación que está teniendo el virus, considero también que es lo más correcto y más seguro para todos. Solo espero y deseo que podamos volver pronto.

Es un sentimiento compartido, otro estudiante expresa: "A la hora de salir, mirábamos el centro mi compañero (...) y yo, dejándolo atrás, sabiendo que, sin querer decirlo, posiblemente no volveríamos". Se suma la preocupación cuando se cierra el primer colegio por posibles casos de COVID-19 en el que hace practicas un compañero. $Y$ otra estudiante escribe:

Mientras me despedía de los niños y las niñas, una niña me ha preguntado que iba a hacer en mi casa estos días y le he dicho que leer y estudiar. Me ha llamado la atención su respuesta ya que me ha dicho "que morro, yo también quiero leer" (...) me ha dicho que en su casa no tiene ningún libro. (...) esas palabras suyas me van a acompañar toda la vida.

Desde la Universitat Jaume I se ofrece la posibilidad de colaborar con las maestras y maestros elaborando materiales para seguir atendiendo al alumnado. A partir de aquí deben elaborar materiales on-line, con una finalidad concreta, a partir de las necesidades detectadas por el profesorado en los colegios, planificando, describiendo y organizando las actividades y, siempre que fuera posible, siguiendo las propuestas de evaluación de los colegios. 


\section{Aguilar-Practicum on-line formación profesorado COVID-19}

Ni la docencia on-line, ni tampoco la normativa general del Practicum de la UJI se adaptan al contexto en pandemia de los centros del prácticum de este artículo. La Comisión de Practicas apoya todas las alternativas que proponemos para paliar este problema.

Desde la UJI se ofrecen ordenadores portátiles al estudiantado. No para todo el mundo es una solución. Una de las estudiantes evidencia el gran problema que ha tenido el estudiantado de la España vaciada: "yo no he podido participar en las videollamadas debido a la mala calidad de la señal del wifi que tenemos actualmente en los pueblos de montaña".

Tampoco todos los contextos son iguales. Una de las estudiantes expresa: "Sigo haciendo malabares para trabajar conciliando la vida familiar con mi hija, no es fácil, pero se aprovecha cada momento de descanso para todo lo contrario, no descansar". Y otra estudiante escribe:

El hecho de estar en un estado de alarma, (...) restricciones sociales y de aislamiento y que además ha puesto en peligro la vida de familiares y amigos muy próximos a mí, ha afectado mi estado anímico, produciendo que me sintiera perdida y angustiada en numerosas ocasiones. (...) Pese a los continuos mensajes alentadores de la comunidad universitaria y de mi tutora de prácticas, los arcoíris como símbolo social y los “todo va a salir bien”, ha habido muchos días en los que he pensado que todas las horas invertidas en este escrito iban a servir para nada y que las prácticas serían pospuestas para el siguiente año. Finalmente, parece que sí que ha servido para algo más que para mantener la mente en otro lugar en estos días tan vacíos y grises.

Como tutora puedo constatar las situaciones complicadas en muchos hogares de mi estudiantado, desde algunas que les han afectado emocionalmente como que sus abuelos estuvieran encerrados solos en sus casas y solo los podían ver por videollamadas, a otras como el fallecimiento en el hospital de familiares sin poderse despedir al estar hospitalizados, y la congoja que les proporcionaba que habían muerto sin tener al lado a su familia, que han afectado profundamente el duelo de los familiares; también padres y madres en paro o con ERTE, afectados por ello, e incluso una intervención quirúrgica de urgencia. A todo se han sobrepuesto y han trabajado con sus maestras y maestros. No ha sido fácil. No solamente el porcentaje de personas de la comunidad universitaria infectadas es un indicador. Los contextos también lo son. 
En algunos casos tras la suspensión de las clases el estudiantado continúa compartiendo lo que les ocurre y una de las tertulias continua adelante online:" Hemos seguido conectados los seis por Skype y hasta hemos hecho Skype para hacer la segunda tertulia".

Pronto descubren sus carencias curriculares. Un estudiante expresa que en las asignaturas de TIC se trabajan muy poco y desconocen algunas herramientas que serían necesarias en el nuevo contexto, señalando:

He aprendido a utilizar herramientas TIC que por desgracia durante la carrera no nos enseñaron a usar, por ejemplo, todas las aplicaciones para hacer conferencias grupales de forma virtual, o un simple editor de videos cómo el que he utilizado para hacer los tutoriales para el alumnado. (...) este periodo me ha ayudado a ser mucho más autodidacta y a preocuparme por descubrir nuevos métodos de enseñanza a distancia, lo cual considero que me ha hecho crecer como futuro docente.

Otra estudiante destaca las múltiples dificultades:

No ha sido fácil adaptar la docencia a la distancia. (...) al no poder comunicarme con ellos, ha sido como trabajar a ciegas, sin saber con qué dificultades se estaban encontrando en cada momento y eso me ha hecho sentir cierta frustración. (...) No es igual programar una unidad didáctica para la enseñanza en el aula que a distancia.

Lo que hacen sus maestras-os supervisores les hace reflexionar. Una estudiante cuenta que cuando unos días antes del confinamiento se comunicó la suspensión de las fiestas de Castelló, que se hubieran celebrado del 14 al 22 de marzo, su maestra se anticipó y preparó una carpeta con actividades de repaso y otra carpeta de "emergencia" por si había confinamiento. Lo gravó en un pendrive y lo repartió. Estaba informada y se anticipó. Había ido explicando a la clase la situación en China y el confinamiento de su población y, cuando llegaron aquí los contagios, les iba informando. La estudiante escribe:

constituyó todo un aprendizaje para mí, ya que yo estaba bastante desinformada de todo. Me di cuenta de lo importante que es en la educación hacer participe al alumnado de los problemas reales de la sociedad y de la 
necesidad de la transversalidad en el aula para que relacionen todo lo que están aprendiendo con las experiencias que viven día a día.

Josep Carles Rius destaca la capacidad de reacción del profesorado para mantener, en la educación a distancia, el vínculo emocional con su alumnado (Rius, 2020):

La pandemia y sus efectos demoledores han puesto aún más el foco en la importancia de la escuela, como espacio de aprendizaje e instrumento de justicia social. El reto de acompañar a los alumnos, a sus familias, y al mismo tiempo intentar mantener la cadena de aprendizajes nos ha hecho redescubrir el extraordinario valor que tiene la escuela a la hora de garantizar la equidad y la justicia social. De compensar las crecientes desigualdades. Sin la escuela, la familia se convierte en el único refugio de los niños y niñas. Pero los docentes saben que para algunos de sus alumnos esto no es así. Porque es la familia la que necesita refugio. Por razones económicas, de vivienda, de vínculos sentimentales, de desconexión con el entorno ... (...) La lucha por la equidad, para combatir la segregación, por la escuela auténticamente inclusiva, será de todos o no será.

El acompañamiento de las maestras y maestros al estudiantado de prácticas aparece reflejado en sus textos. El papel de este profesorado en un contexto en el que la mayoría de familias ya estaban afectadas por la brecha digital, en un contexto familiar que no favorece el estudio, con muchas familias iletradas que, en muchísimos casos, no disponen de ningún acceso digital a la información, es muy importante. Muchas de estas familias han crecido en un contexto en el que no han tenido ninguna expectativa educativa. El número de personas que conviven en la misma casa y las condiciones de las mismas, tampoco favorecen un espacio de estudio. La situación es muy complicada. Una estudiante explica:

En el momento de inicio de la pandemia, muchos tutores están incomunicados con ellos [alumnado] y no pueden ejercer la docencia on-line (...) mi maestra ha conseguido por su cuenta contactar con los alumnos y realizar diferentes videollamadas y encuentros on-line. 
Otra estudiante recoge en su texto:

los maestros-as no solo se han dedicado a adaptar sus intervenciones, cambiar las planificaciones previstas, mandar tareas y corregir estas, seguir con las reuniones $(\ldots)$... sino que también a través del grupo de mensajería instantánea donde está toda la comunidad educativa, además de informar de las novedades, han mandado fotografías suyas o vídeos protagonizados por ellos mismos-as con un toque de humor para animar a los niños/as y a los familiares.

Pero enviarlo, recibirlo y poder seguir las tareas, son cosas diferentes. Una estudiante escribe:

La mayoría de las familias han agradecido mucho que les llamemos y preguntemos cómo están. Han visto que nos preocupamos y este contacto, ha hecho que (...) se interesen un poco más por las tareas. [la maestra] ha estado subiendo contenido semanal a un blog (...) la mayoría no tienen libretas y lápices para hacer las actividades manualmente.

Una estudiante que hace su prácticum en la CdA narra que la segunda semana del confinamiento la maestra contacta telefónicamente con las familias para ver si habían podido acceder al PDF de actividades y las familias responden que acceden a internet por megas desde el móvil, que no tienen impresoras, y que no sabían nada del PDF de actividades. La maestra contacta con una madre que va a coordinar un grupo de whatsapp con todas las familias. Esta madre se involucró de pleno. La séptima semana empiezan a hacer conferencias con las familias para que el alumnado se viera, esto señala la estudiante, "hizo que se incrementase la participación de las familias en las actividades”. En otro de los colegios las maestras llaman uno a uno a su alumnado, una estudiante narra:

al principio estaban prácticamente ausentes [alumnado], pero poco a poco fueron mandando videos de como realizaban las actividades, (...) provocaba bastante emoción. Así se fueron sumando cada vez más alumnos a las actividades hasta que, al final, las realizaban prácticamente todos y todas. Todo este proceso me ha hecho reflexionar bastante, creo que las leyes, las normas, los consejos que han estado facilitando los medios relacionadas con la educación, estaban pensadas para realizarlas en un tipo de colegio y de 
alumnado con unas situaciones personales muy diferentes a las del [CAES]. Si hubiéramos hecho lo mismo que hacían otras compañeras mías que estaban en otros colegios, es decir las fichas del principio, seguramente el alumnado a día de hoy seguiría ausente. Creo que esto es un claro ejemplo de la importancia de contextualizar la enseñanza.

Una de las estudiantes destaca que, en su escuela, el ayuntamiento de Castelló y la escuela proporcionaron tabletas táctiles con internet a las familias que no tienen recursos digitales y/o conexión a internet. Otra estudiante explica que en uno de los colegios se ha utilizado un grupo de "telegram" durante la cuarentena:

para enviar vídeos de ánimo por la situación actual, instrucciones para aquellos que tengan que canjear el vale del comedor, las tareas para que los niños las hagan en casa, información o actividades de interés para hacer durante el tiempo del confinamiento.

Sin embargo, otra estudiante de otra escuela escribe: "Las familias del aula carecen de competencia digital (...) Ninguna (...) disponía de impresora.” Y otra estudiante cuenta:

Nos comentaron que les costaba mucho descargarse los vídeos largos que les enviábamos vía telegram, así que creímos que una poción alternativa eficaz sería subirlos a la plataforma de youtube y enviarles el link. Esta alternativa dio buenos resultados.

Es complicado trabajar, otra estudiante escribe sobre la falta de medios y como los maestros y maestras se acercan a las casas:

es una odisea tanto para el profesorado como para el alumnado, cuando no dispone de dispositivos o recursos tecnológicos (...) Este inconveniente no les deja avanzar en su formación, los profesores/as acuden semanalmente en muchos casos a los domicilios de estos alumnos-as para entregar, recoger y corregir el material preparado. (...) Este material se puede hacer con lápiz y papel. Posteriormente mandan una foto del trabajo realizado siempre que se disponga de acceso a un móvil de los familiares del alumnado o se busca otra alternativa, siendo los mismos profesores/as quienes recogen el material. 
La situación en otro de los colegios es similar, otra estudiante recoge que puesto que la brecha digital es una realidad:

El colegio ha buscado una respuesta (...) el director ha repartido [tarea para hacer] por las casas de los niños para que sigan adelante y no pierdan el curso. Ya que las clases no pueden llevarse a cabo, (...) muchas familias no tienen acceso a internet, ni los recursos necesarios (...) Por tanto mi supervisora se puso en contacto conmigo para explicarme la situación, como iba a seguir adelante el curso y he ido haciendo lo que se me iba pidiendo.(...) Siempre he estado en contacto con la supervisora[maestra] mediante llamada, WhatsApp o correo electrónico, y me lo ha puesto todo muy fácil,(...) si he tenido alguna duda siempre ha estado ahí para resolverla. Ha sido fascinante ver cómo se ha adaptado todo, [para] no perder el contacto, y que las familias siempre puedan estar en contacto con las maestras y los maestros, para cualquier duda. (...) las maestras también llaman a las familias para ver cómo va todo y si los niños están realizando la faena. He podido asistir a reuniones de ciclo y de inclusión, ha sido una manera de estar informada, de escuchar. De aprender, de ver diferentes opiniones y sobre todo ha sido una forma distinta de hacer estas prácticas y ver cómo sigue adelante el curso a pesar de la situación (...) Se trabaja mediante la aplicación Discord. (...) los maestros y maestras no dejan de trabajar para que los niños no pierdan el curso, trabajen y les tengan de alguna manera cerca. (...) me ha hecho muy feliz ver que lo que hacía valía la pena, ser de ayuda y ver el gran trabajo realizado por todas las maestras.

Mercè Gisbert, experta en tecnología educativa, expone en una entrevista las diferencias entre virtualidad y presencialidad que se obvian (Gisbert, 2020):

"La virtualización no significa hacer la misma clase presencial de cada día por videoconferencia y, aún menos, pretender pasar lista". Esto no es virtualizar la docencia, "esto es hacer presencial la virtualidad, que es todo lo contrario", apunta la investigadora. En cuanto a la Universidad, propone que, además de ser conscientes de lo que se quiere conseguir, hay que confiar en los estudiantes, saber que se les deben marcar unos objetivos, unas actividades y unas fechas límite que ellos deberán cumplir y asumir sus responsabilidades, y que no hacerlo tendrá unas repercusiones igual que las tiene en la presencialidad. [...] El modelo de formación presencial que hemos desarrollado hasta ahora no podemos encapsularlo en videoconferencias y quedarnos aquí pensando que ya lo tenemos todo a punto", ya que esto puede 


\section{Aguilar-Practicum on-line formación profesorado COVID-19}

funcionar de momento "pero a corto plazo nos traerá muchos inconvenientes y será muy poco eficiente", explica. Hay que ser conscientes de que no se puede concebir "un busto parlante durante una hora, como los telediarios hace unos años".

En relación a la virtualidad una estudiante expresa su vivencia:

Algo que deja claro este periodo de actividad no presencial es que el trabajo diario del docente en clase es insustituible, no existe ningún método virtual que pueda reemplazar tener a los alumnos cerca, tener conversaciones cara a cara, poder aconsejar, guiar, responder, etc. Esta relación que se forma entre docente y alumnado durante la actividad presencial, es la mayor parte de la educación y nunca podremos sustituirla por blogs, videos o actividades online. Y desde luego menos aún en este tipo de centros, dónde lo importante es el día a día. Las relaciones que se generan y las diferentes situaciones que se dan dentro del aula.

Otra estudiante refleja que ha buscado algo positivo en la experiencia, a pesar de todo:

No se puede comparar el punto de vista del aula y el punto de vista desde casa. No me ha sido satisfactorio terminar mis primeras prácticas así. Lo único positivo que encuentro es que he podido trabajar con una maestra de escuela, para elaborar materiales para que les llegasen a los niños-as o que familiares agradeciesen el trabajo hecho por nosotros y que estuvieran contentos con ello.

En relación a las maestras y los maestros, una estudiante destaca el compromiso educativo:

Vi algo más que simples trabajadores, vi gente comprometida, vi esfuerzo y, sobre todo vi personas implicadas en facilitar todas las herramientas que tienen en sus manos para que niños que no han nacido en un contexto favorable puedan utilizarlas para "construir un futuro diferente".

Y otra estudiante escribe: "Hemos estado aislados durante dos meses sin poder salir a la calle en muchos casos, pero esto no ha sido un inconveniente porque hemos estado más conectados y vivos que nunca". 


\section{No mirar hacia otro lado}

Patricia Martínez defiende que los cierres masivos de las instalaciones escolares en todo el sistema educativo han evidenciado tanto su importancia como sus debilidades estructurales, pero, además, se ha vuelto a considerar "como un ecosistema que nos interconecta a todos (Martínez, 2020). En España, destaca Cristina Monge (2020), la inversión en formación del profesorado está por debajo de lo que se dedicaba en 1997 y en investigación educativa las cifras nos sitúan en los niveles de 2005. Consecuentemente, defiende que este el momento para replantear en todo el sistema educativo las formas de educar, y subraya que en la Universidad no debe perderse el tiempo en lecciones magistrales que pueden encontrarse en la red, y en ocasiones mucho mejores, porque el tiempo debemos emplearlo en fomentar la discusión, el trabajo en equipo y el pensamiento crítico.

Debemos no olvidar, como subraya Enrique Diez (2020c), que "la revolución educativa pendiente no es tecnológica, sino inclusiva". El contenido de este artículo es uno de los ejemplos de que, efectivamente, lo es. Y las medidas que se toman para lograrlo pasan, necesariamente, por la inversión. Evidenciando la necesidad de duplicar el número de profesorado, además de acondicionar y ampliar espacios, destacando: “. Si no hay más maestras, maestros, profesoras y profesores suficientes en todos los niveles educativos, no servirá de nada todos los esfuerzos que hagamos. Obviamente, estas medidas, señala, deberán incluir una serie de cambios concretos que deben producirse simultáneamente (Diez, 2020c).

Si esto no se lleva a la práctica, argumenta la razón será ideológica, en lugar de buscar el bien común, aplicar el ahorro de costes neoliberal que lleva a que la mayoría de la ciudadanía pierda cada vez más derechos sociales y vitales, destacando que sí que existen medios y recursos, pero no es la educación la que se prioriza (Diez, 2020c).

En nuestro contexto existen realidades marcadas por la desigualdad económica y de recursos, el informe para luchar contra la pobreza elaborado por Philip Alston (2020), el relator de la ONU, que visitó España a principios de 2020, ha visibilizado que se concentran en escuelas segregadas el $44 \%$ de los estudiantes y el $72 \%$ de niñas y niños en situaciones vulnerables, principalmente romaníes y migrantes y, además, muestra las consecuencias de esta desigualdad como, por ejemplo, el abandono escolar o 


\section{Aguilar-Practicum on-line formación profesorado COVID-19}

las distintas expectativas de futuro educativo $\mathrm{y}$, consecuentemente, de realidades vitales .

La Asociación Secretariado General Gitano evidencia que en la concertada hay entre un 7 y un $10 \%$. Mayoritariamente, señala también, están en la pública e incide en algo muy importante en relación a esta situación (ASG, 2020):

el subvencionismo a la escuela privada y el sistema de admisión de alumnos lleva a la conformación de escuelas específicas, donde a la vez que se van matriculando niños y niñas gitanos $y$ no gitanos de familias socioeconómicamente de privadas y marginales, se van marchando los no gitanos y los gitanos con más interés en la escuela a otras escuelas (concertadas o públicas sin alumnado marginal) (...)

Lo vivido durante el confinamiento, por el estudiantado de formación inicial de maestra-o, en estos cuatro colegios, demuestra que esa realidad es, en demasiadas ocasiones, ajena al currículum universitario y a las normativas del prácticum que homogeneizan la realidad escolar. La realidad es que, cuando el estudiantado de prácticum llega a las escuelas, poco o nada conocen de la cultura del alumnado de las mismas, niñas y niños gitanos-as que, a la vez, son valencianos-as.

Una encuesta de la Fundación Secretariado Gitano, realizada a once mil participantes en sus programas, para conocer cómo está afectando la pandemia al colectivo gitano, informaba en junio de 2020 de lo siguiente ( $\mathrm{La}$ Voz, 2020)

Más del $40 \%$ de las personas de etnia gitana han tenido problemas de acceso a la alimentación durante la crisis sanitaria. La razón: no tener dinero para comprar alimentos. Es uno de los resultados de El $64 \%$ de los encuestados ya estaban desempleados antes de la crisis sanitaria: solo el $14 \%$ estaba trabajando por cuenta ajena, el $3 \%$ por cuenta propia en la venta ambulante y un $19 \%$ en la venta ambulante no regularizada o en la chatarra. Una situación de desigualdad y vulnerabilidad que se ha acrecentado durante la pandemia. El $34 \%$ de los encuestados tiene dificultades para pagar la luz, el $31 \%$ el agua, el 25,6\% el alquiler y el $25 \%$ el gas. La situación laboral de dos de cada tres personas gitanas asalariadas ha empeorado: más de un tercio ha perdido su trabajo, otro tercio ha sido incluido en un ERTE y a un $12 \%$ le han reducido la jornada. 
El $37 \%$ cree que se les está estigmatizando, ofreciendo una imagen negativa e injusta sobre la comunidad gitana en el contexto de la crisis del COVID-19.

Es necesario recuperar la voz al estudiantado de prácticas, no podemos olvidarla en la reflexión sobre sus prácticas en las escuelas, en su experiencia como futuros maestros y maestras. No se pueden diseñar unas prácticas ajenas a la realidad de los contextos donde el estudiantado de formación inicial de maestra-a va a realizarlas, conocer esa realidad desde su experiencia protagonista es muy importante. Las voces del estudiantado muestran que ha habido maestras y maestros, anónimos (no han salido en los medios de comunicación), comprometidos con su alumnado, que han buscado alternativas en contextos sociales y educativos muy complicados, que frente a la educación on-line prescrita siempre que fuera posible, han llevado puerta a puerta las tareas, para poder seguir adelante porque era la única alternativa viable. También que ha habido familias que se han comprometido para que sus hijas e hijos no se quedaran sin educación escolar, desde los escasos medios con los que contaban, como la madre coordinadora del grupo de WhatsApp que dinamiza al resto de madres, potenciando el sentimiento de comunidad educativa. A pesar de todo este esfuerzo, se ha evidenciado la carencia de formación y de recursos que ha dificultado en contextos de exclusión social, tanto al profesorado como a las familias de las escuelas y, también, al profesorado universitario responsable de las prácticas, poder seguir adelante sin dejar a nadie atrás. Contextos unidos a la brecha digital, social y educativa que ya existían antes de la pandemia, no se puede ignorar, y que se ha agudizado en un contexto de COVID-19, en la problemática del trabajo en el día a día.

Ojalá en el futuro no siga ocurriendo. Está visibilizado, solamente debemos solucionarlo, no es cuestionable. Y es urgente en este contexto, para que nadie, absolutamente nadie, se quede atrás.

Las conclusiones de esta experiencia de prácticum en pandemia, se suman, desde lo que se ha vivido, a lo que las investigaciones citadas a lo largo del artículo han aconsejado:

1) La necesidad de una revisión curricular crítica que incluya aquellos contenidos realmente relevantes y, además, incluir el contexto del alumnado. 
94 Aguilar-Practicum on-line formación profesorado COVID-19

2) La necesidad de una revisión de la evaluación, como defiende Jan McArthur (2019), como un proceso ético extenso en el que tanto profesorado como alumnado, desafíen las prácticas aceptadas, buscando nuevas alternativas y posibilidades, desde una evaluación entendida como una cuestión de justicia social.

3) La necesidad de formar en TIC al profesorado de las escuelas, al estudiantado de formación inicial de maestro-a y a los tutores-as del prácticum de la universidad, para que dispongan de herramientas suficientes y adecuadas para la docencia virtual, pudiendo elegir la más adecuada en cada ocasión.

\section{Referencias}

Agencia Europa Press. (2020, julio 24). Uno de cada tres niños de Primaria no recibió clases online durante el confinamiento, según OCU.

Escuela.

http://www.periodicoescuela.es/Content/Documento.aspx? params=H4s IAAAAAAAEAMtMSbH1czUwMDAyNDcyMzRUK0stKs7Mz7M1 MjAyMDA3MlHLy09JDXFxti3NS01Ny8xLTQEpyUyrdMlPDqksSL VNS8wpTIVLTcrPz0YxKR5mAgCNgShpYwAAAA==WKE

Aguilar, C. (2012). Los diarios dialógicos del estudiantado en el prácticum:

la tinta de la vida" En Fernández, E., \& Rueda, E. (coords.). La educación como elemento de transformación social. Libro de Actas del XII Congreso Internacional de Formación del Profesorado (pp. 897906). Asociación Interuniversitaria de Formación del Profesorado (AUFOP)/Universidad de Valladolid/ GEEPP Ediciones. http://repositori.uji.es/xmlui/handle/10234/72686

Aguilar, C. (2017). La tertulia pedagógica dialógica en el prácticum de la formación inicial de maestras y maestros. Revista Iberoamericana de Educación, 73(2), 9-22. https://doi.org/10.35362/rie732198

Alston, P. (2020). Declaración del Relator Especial de las Naciones Unidas sobre la extrema pobreza y los derechos humanos, Philip Alston, sobre la conclusión de su visita oficial a España, 27 de enero - 7 de febrero de 2020. Naciones Unidas. Derechos Humanos. Oficina del Alto Comisionado.

https://www.ohchr.org/SP/NewsEvents/Pages/DisplayNews.aspx?New $\mathrm{sID}=25524 \&$ LangID $=\mathrm{S}$ 
Asociación Secretariado General Gitano (2020, diciembre). El pueblo gitano y la educación. Aula Intercultural, $7 / 8$.

https://aulaintercultural.org/2000/12/07/el-pueblo-gitano-y-laeducacion/

Díez, E. (2020a, julio 14). Releer a Freire en tiempos de pandemia. El diario de la educación.

https://eldiariodelaeducacion.com/2020/07/14/releer-a-freire-entiempos-de-pandemia/

Díez E. (2020b, julio 16). La pandemia neoliberal el común.es. https://elcomun.es/2020/06/16/la-pandemia-neoliberal/

Diez, E (2020c, julio 2). Bajar la ratio y aumentar la plantilla docente: plan de reconstrucción educativa. el diario de la educación. https://eldiariodelaeducacion.com/2020/06/02/bajar-la-ratio-yaumentar-la-plantilla-docente-plan-de-reconstruccion-educativa/

Diez, E., \& Gajardo, K. (2020a). Educar y Evaluar en Tiempos de Coronavirus: la Situación en España. Multidisciplinary Journal of Educational Research, 10(2), 102-134.

Diez, J., \& Gajardo, K. (2020b). Políticas Educativas en Tiempos de Coronavirus: La Confrontación Ideológica en España. Revista Internacional de Educación para la Justicia Social, 2020, 9(3e), 83101. https://doi.org/10.15366/riejs2020.9.3.005

Diez, E., \& Torrego, L. (2020). "Hablemos de educación. ep 3 - enrique díez y luis Torrego". Editorial Octaedro. 12 de mayo.

https://www.youtube.com/watch?v=Ps0OqcNPvM4\&feature=youtu.be

EAPN. (2020, julio 16). EAPN-ES analiza el perfil y mapa de la pobreza severa de la última década en España.

https://eapn.es/noticias/1 180/eapn-es-analiza-el-perfil-y-mapa-de-lapobreza-severa-de-la-ultima-decada-en-espana

Gisbert, M. (2020, marzo 24). Hacer virtual la docencia no debe ser hacer presencial la virtualidad.URVactiv@.

https://diaridigital.urv.cat/es/hacer-virtual-la-docencia-no-debe-serhacer-presencial-la-virtualidad/

Gutierrez, P. (2020a, mayo 12). Save the Children reclama 4.200 millones para el sistema educativo". El diario de la educación. https://eldiariodelaeducacion.com/2020/05/12/save-the-childrenreclama-4-200-millones-para-el-sistema-educativo/ 
La Voz. (2020, junio 5). Una de cada tres personas de etnia gitana tiene problemas para pagar los recibos. La Voz de Galicia. https://www.lavozdegalicia.es/noticia/ferrol/ferrol/2020/06/05/trespersonas-etnia-gitana-problemas-pagarrecibos/0003_202006F5C2992.htm

Martinez, P. (2020, junio 20). Conocimiento compartido para no dejar a nadie atrás. Ctxt, https://ctxt.es/es/20200601/Firmas/32587/agenda2030-universidad-ciencia-abierta-COVID19-pastora-martinezsamper.htm

McArthur, Jan. (2019). La evaluación: una cuestión de justicia social. Perspectiva crítica y prácticas adecuadas. Madrid: Narcea.

Monge, C. (2020, junio 15). Acordarse de la escuela cuando cierra. Infolibre.es.

https://www.infolibre.es/noticias/opinion/columnas/2020/06/15/acordar se_escuela_cuando_cierra_107752_1023.html

Redacción (2020, julio 10). Cuando un tercio del alumnado se queda descolgado. El Diario de la Educación.

https://eldiariodelaeducacion.com/2020/07/10/cuando-un-tercio-delalumnado-se-queda-descolgado/

Rius, J.C. (2020, julio 1). Elogi dels docents, que han lluitat pels seus alumnes. El diari de l'educació. https://diarieducacio.cat/elogi-delsdocents-que-han-lluitat-pels-seus-alumnes/

Sancho, J. (2020, junio 21) Els qui afirmen que la pandèmia ha revelat la desigualtat... de debò no ho sabien?. El diari de l'educació. https://diarieducacio.cat/els-qui-afirmen-que-la-pandemia-ha-revelatla-desigualtat-de-debo-no-ho-sabien/

Torrego, L. (2020, junio 15). ¿El COVID-19 ha traído la desigualdad a la educación?. Concejo Educativo - Movimiento de Renovación Pedagógica de Castilla y León. http://www.concejoeducativo.org/2020/el-COVID-19-ha-traido-ladesigualdad-a-la-educacion/

Consol Aguilar Ródenas es Catedrática de Escuela Universitaria del Departament de Pedagogia i Didàctica de les CC. Socials, la Llengua i la Literatura de la Universitat Jaume I (España)

Contact address: aguilar@uji.es 